\title{
Association of ventricular arrhythmias with left ventricular remodelling after myocardial infarction
}

Aleksandar D Popović, Aleksandar N Nešković, Kočo Pavlovski, Jelena Marinković, Rade Babić, Milovan Bojić, Ming Tan, James D Thomas

\begin{abstract}
Objective-To assess the relation between ventricular arrhythmias after myocardial infarction and left ventricular remodelling.

Design-Prospective study with consecutive patients.

Methods -97 patients with acute myocardial infarction underwent serial echocardiographic examinations (days $1,2,3$, and 7 , and after 3 weeks) to determine end diastolic volume, end systolic volume, and ejection fraction; volumes were normalised for body surface area and expressed as indices. Holter monitoring was performed on the day of the final echocardiogram. Coronary angiography was performed in 88 patients before hospital discharge.

Results-Complex ventricular arrhythmias (defined as Lown class 3-5) were found in 16 of 97 patients. In logistic regression models, variables predictive of complex ventricular arrhythmias were end systolic volume index on admission $(b=0.054, P=0.015)$ and end diastolic volume index after three weeks $(b=$ $0.034, P=0.012)$. Complex arrhythmias were also related to the increase of end diastolic and end systolic volume indices throughout the study $(F=5.62, P=$ 0.046 and $F=6.42, P=0.017$, respectively by maNOVA). A two stage linear regression model of ventricular volume versus time from infarct showed that both intercept (initial volume) and slope (rate of increase) were higher for patients with complex arrhythmias in both diastole and systole $(P<0.001$ for all $)$.

Conclusions-Complex ventricular arrhythmias after myocardial infarction are related to the increase of left ventricular volume rather than to depressed ejection fraction. Complex arrhythmias may be an aetiological factor linking left ventricular remodelling with higher mortality, but larger follow up studies of patients with progressive left ventricular dilatation after myocardial infarction are necessary to answer these questions.
\end{abstract}

(Heart 1997;77:423-427)

Keywords: myocardial infarction; left ventricular remodelling; ventricular arrhythmias
It is well known that both ventricular arrhythmias and left ventricular dysfunction after myocardial infarction are predictors of poor prognosis, identifying patients who are at increased risk of death. ${ }^{1}$ Following acute myocardial infarction there are complex changes in ventricular architecture ${ }^{2}$ in both infarct and non-infarct zones, a process known as left ventricular remodelling. A critical change is that of infarct expansion, ${ }^{2}$ in which disruption of normal myofibrils leads to thinning and dilatation of the necrotic zone. We have recently shown that left ventricular dilatation can be detected within hours after infarction and that it can be prevented by thrombolytic treatment. ${ }^{3}$ On the other hand, several studies ${ }^{14}$ have assessed the relation between ventricular arrhythmias and left ventricular ejection fraction, with controversial results; thus the relation between ventricular arrhythmias and left ventricular remodelling after myocardial infarction has not been clarified.

The aim of this study was to assess the relation between ventricular arrhythmias after myocardial infarction and relevant clinical, echocardiographic, and angiographic findings and, particularly, to investigate the association of ventricular arrhythmias with early left ventricular remodelling, as assessed by serial echocardiographic examinations.

\section{Methods}

STUDY PATIENTS

We prospectively evaluated 97 consecutive patients with first acute myocardial infarction who met the following criteria: age 70 years, chest pain lasting longer than 30 minutes, ST segment elevation $2 \mathrm{~mm}$ at least in two electrocardiographic leads, transient elevation of creatine kinase and/or $\mathrm{MB}$ isoenzyme, echocardiogram performed within 24 hours of the onset of pain, and Holter monitoring performed after three weeks.

\section{TREATMENT}

Thrombolytic treatment (intravenous streptokinase 1500000 units over 30 to $60 \mathrm{~min}$ utes) was given in 58 patients. The remaining 39 patients did not receive thrombolytic treatment at the discretion of their attending cardiologist, because they were admitted late after the onset of pain or they had contraindication for thrombolysis. 
ECHOCARDIOGRAMS

Complete cross sectional and Doppler echocardiograms were performed within 24 hours of the onset of pain, on days $2,3,7$, and after three weeks in order to determine end diastolic volume, end systolic volume, and ejection fraction. All examinations were performed with an Acuson 128 machine (Mountain View, California, USA), using a $2.5 \mathrm{MHz}$ transducer; they were stored on $\frac{1}{2}$ inch VHS videotapes for later analysis. Left ventricular end diastolic volume, end systolic volume, and ejection fraction were determined from apical two and four chamber views using the Simpson's biplane formula, according to the recommendations of the American Society of Echocardiography. ${ }^{5}$ Tracing of endocardial borders in end diastole and end systole was performed on the Acuson 128 machine in the technically best cardiac cycle. The volumes were normalised for body surface area and expressed as indices. Echocardiographic measurements were performed by a single observer (ANN) who was blinded to clinical, angiographic, and Holter data.

HOLTER MONITORING

Two channel 24 hour Holter monitoring (De Mar, model 152, Irvine, California, USA) was performed three weeks after infarction (on the day of the final echocardiogram). Antiarrhythmic agents were withheld for 48 hours before Holter monitoring. Ventricular arrhythmias were classified using the Lown classification $^{6}$ and patients were grouped into those with simple ventricular arrhythmias (Lown class 0 to 2 ) and patients with complex ventricular arrhythmias (Lown class 3 to 5 ). ${ }^{4}$

\section{CORONARY ANGIOGRAPHY}

Coronary angiography was performed before hospital discharge in 88 patients and perfusion of the infarct related artery was assessed using

Table 1 Incidence of ventricular arrhythmias

\begin{tabular}{|c|c|c|c|c|}
\hline Lown class $^{6}$ & $\begin{array}{l}\text { No of } \\
\text { patients } \\
(n=97)\end{array}$ & $\begin{array}{l}\text { No of PVCs/ } \\
24 h \\
(n=97)\end{array}$ & $\begin{array}{l}\text { No of couplets/ } \\
24 h \\
(n=8)\end{array}$ & $\begin{array}{l}\text { No of NSVTs/ } \\
24 h \\
(n=3)\end{array}$ \\
\hline 0 (No PVCs) & 24 & 0 & - & - \\
\hline 1 (Occasional PVCs) & 51 & $62(121)$ & - & - \\
\hline 2 (Frequent PVCs; > 30/h) & 6 & $1028(620)$ & - & - \\
\hline 3 (Multiform PVCs) & 6 & $703(1084)$ & - & - \\
\hline 4 (Repetitive PVCs) & 10 & $456(523)$ & $4 \cdot 3(4 \cdot 3)$ & $1 \cdot 7(0 \cdot 6)$ \\
\hline 5 (Early PVCs) & 0 & - & & \\
\hline
\end{tabular}

PVC, premature ventricular contraction; NSVT, non-sustained ventricular tachycardia. Values are expressed as mean (SD).

Table 2 Patient demographics

\begin{tabular}{llll}
\hline & $\begin{array}{l}\text { Total } \\
(n=97)\end{array}$ & $\begin{array}{l}\text { Arrhythmias } \\
(n=16)\end{array}$ & $\begin{array}{l}\text { Arrhythmias }- \\
(n=81)\end{array}$ \\
\hline Age, years & $54 \cdot 8(9 \cdot 7)$ & $57 \cdot 3(6 \cdot 9)$ & $54 \cdot 4(93)$ \\
Sex & $22 \mathrm{~F} / 75 \mathrm{M}$ & $3 \mathrm{~F} / 13 \mathrm{M}$ & $19 \mathrm{~F} / 62 \mathrm{M}$ \\
Hypertension & $36 / 97$ & $3 / 16$ & $33 / 81$ \\
Diabetes & $14 / 97$ & $0 / 16$ & $14 / 81$ \\
Smoking & $54 / 97$ & $7 / 16$ & $47 / 81$ \\
Anterior infarct & $39 / 97$ & $8 / 16$ & $13 / 81$ \\
Killip class $>1$ & $18 / 97$ & $5 / 16$ & $1019(647) \dagger$ \\
CK level (U/1) & $1133(814)$ & $1709(1258)$ & $47 / 81$ \\
Thrombolysis & $58 / 97$ & $11 / 16$ & $38 / 74$ \\
Patent IRA & $46 / 88$ & $8 / 14$ & $34 / 74$ \\
Multivessel CAD & $40 / 88$ & $6 / 14$ & \\
\hline
\end{tabular}

Arrhythmias +, patients with complex ventricular arrhythmias; Arrhythmias -, patients without complex ventricular arrhythmias; $C A D$, coronary artery disease; $C K$, peak creatine kinase level; IRA, infarct related artery. Values for age and CK are expressed as mean (SD).

$+P<0.01$.
TIMI criteria. ${ }^{7}$ It was not performed in nine patients who refused. Significant stenosis was defined as $>70 \%$ stenosis of the major coronary artery. Successful reperfusion, defined as TIMI grade 3, was achieved in 45 of 88 patients.

\section{STATISTICAL ANALYSIS}

To test for differences between patients with and without complex arrhythmias, we used the unpaired $t$ test for continuous variables and the $\chi^{2}$ test for categorical variables. Logistic regression was used to identify variables predictive of complex ventricular arrhythmias, and repeated measures analysis of variance was used to assess the association of left ventricular dilatation with ventricular arrhythmias. To have a more uniform temporal sampling to observe volume change, time was expressed as the base- 2 logarithm (day $1=0$, day $2=1$, day $21=4 \cdot 34)$. A two stage linear regression model that was described previously $^{3}$ was therefore used to analyse temporal changes of left ventricular end diastolic volume and end systolic volume indices. The volumes of each patient over time were analysed by a linear regression model with random intercept and slope. The intercepts and slopes among patients with and without complex arrhythmias were then compared using the Wald test through an overall random effects model.$^{8}$

\section{Results}

Complex ventricular arrhythmias (Lown class 3 to 5$)^{4}$ were found in 16 patients (multiform premature ventricular contractions (PVC) in six, and repetitive PVC in 10). Among patients with repetitive PVC, couplets were detected in eight patients, non-sustained ventricular tachycardia in two patients, and both arrhythmias in one patient (table 1). In three patients with non-sustained ventricular tachycardia, there were five episodes of tachycardia, ranging from four to eight beats.

There was no significant difference in age, sex, history of hypertension, diabetes or cigarette smoking, infarct location, Killip class, use

Table 3 Predictors of complex ventricular arrhythmias on admission

\begin{tabular}{ll}
\hline & Significance \\
\hline Infarct location & $\mathrm{P}=0.96$ \\
Killip class & $\mathrm{P}=0.76$ \\
Use of thrombolytic treatment & $\mathrm{P}=0.14$ \\
End diastolic volume index on admission & $\mathrm{P}=0.82$ \\
End systolic volume index on admission & $\mathrm{P}=0.015$ \\
Ejection fraction on admission & $\mathrm{P}=0.38$ \\
\hline
\end{tabular}

Table 4 Predictors of complex ventricular arrhythmias at three weeks

\begin{tabular}{ll}
\hline & Significance \\
\hline Infarct location & $P=0.77$ \\
Killip class & $P=0.51$ \\
Use of thrombolytic treatment & $P=0.15$ \\
Patency of the infarct related artery & $P=0.30$ \\
Extent of coronary artery disease & $P=0.92$ \\
End diastolic volume index at 3 weeks & $P=0.012$ \\
End systolic volume index at 3 weeks & $P=0.79$ \\
Ejection fraction at 3 weeks & $P=0.83$ \\
\hline
\end{tabular}


Table 5 Left ventricular volumes in patients with and without complex ventricular arrhythmias

\begin{tabular}{llllll}
\hline & \multicolumn{2}{l}{ End diastolic volume index $\left(\mathrm{ml} / \mathrm{m}^{2}\right)$} & & \multicolumn{2}{l}{ End systolic volume index $\left(\mathrm{ml} / \mathrm{m}^{2}\right)$} \\
\cline { 2 - 3 } \cline { 5 - 6 } \cline { 5 - 6 } & Arrhythmias + & Arrhythmias & & Arrhythmias + & Arrhythmias - \\
\hline Day 1 & $67 \cdot 6(4 \cdot 0)$ & $61 \cdot 1(1 \cdot 4)$ & & $40 \cdot 4(3 \cdot 3)$ & $32 \cdot 7(1 \cdot 3)^{\star}$ \\
Day 2 & $73 \cdot 3(5 \cdot 1)$ & $62 \cdot 5(1 \cdot 5) \dagger$ & & $43 \cdot 4(4 \cdot 2)$ & $33 \cdot 1(14) \dagger$ \\
Day 3 & $74 \cdot 0(5 \cdot 2)$ & $64 \cdot 4(1 \cdot 7)^{\star}$ & & $43 \cdot 7(4 \cdot 6)$ & $34 \cdot 2(1 \cdot 6)^{\star}$ \\
Day 7 & $75 \cdot 1(5 \cdot 4)$ & $66 \cdot 1(1 \cdot 9)$ & & $43 \cdot 9(4 \cdot 3)$ & $35 \cdot 8(1 \cdot 7)$ \\
Day 21 & $80 \cdot 8(6 \cdot 3)$ & $67 \cdot 1(2 \cdot 1)^{\star}$ & & $47 \cdot 8(5 \cdot 3)$ & $35 \cdot 7(1 \cdot 9)^{\star}$ \\
\hline
\end{tabular}

Arrhythmias +, patients with complex ventricular arrhythmias; Arrhythmias -, patients without complex ventricular arrhythmias. Values are expressed as mean (SEM).

$\star \mathrm{P}<0.05 ;+\mathrm{P}<0.01$.

Table 6 Temporal analysis of left ventricular volumes in patients with and without complex ventricular arrhythmias

\begin{tabular}{|c|c|c|c|c|}
\hline & \multicolumn{2}{|c|}{ End diastolic volume index $\left(\mathrm{ml} / \mathrm{m}^{2}\right)$} & \multicolumn{2}{|c|}{ End systolic volume index $\left(\mathrm{ml} / \mathrm{m}^{2}\right)$} \\
\hline & Arrhythmias + & Arrhythmias- & Arrhythmias + & Arrhythmias- \\
\hline Intercept & $\begin{array}{r}68.7(3.2) \\
2 \cdot 8(0.7)\end{array}$ & $\begin{array}{r}61.3(1.4) \ddagger \\
1.4(0.3) \pm\end{array}$ & $\begin{array}{r}42.6(2.9) \\
1.6(0.6)\end{array}$ & $32 \cdot 8(1 \cdot 3) \ddagger$ \\
\hline
\end{tabular}

Arrhythmias + , patients with complex ventricular arrhythmias; Arrhythmias - , patients without $\ddagger \mathrm{P}<0.001$.
complex ventricular arrhythmias. Values are expressed as mean (SEM).

Figure 1 Association of complex ventricular arrhythmias with end arrhythmias with end
diastolic volume changes. Arrhythmias + , patients with complex ventricular arrhythmias; Arrhythmias -, patients without complex ventricular arrhythmias; EDVi, end diastolic volume index. ${ }^{\star} P<0.05 ;+P<0.01$ of thrombolysis, patency of the infarct related artery, and the angiographic extent of coronary artery disease between patients with and without complex arrhythmias; however, peak creatine kinase values were higher $(P<0.05)$ in patients with complex ventricular arrhythmias (table 2).

When complex ventricular arrhythmias (as a dependent variable) were entered into the logistic regression model with infarct location, Killip class, use of thrombolytic treatment, end diastolic volume index, end systolic volume index, and ejection fraction on admission (as independent variables), the only variable predictive of complex ventricular arrhythmias was end systolic volume index $(b=0.054$, $P=0.015$ ) (table 3). Similar findings were observed after three weeks with the regression model that included patency of the infarct related artery and the angiographic extent of coronary artery disease (table 4 ): the only variable predictive of ventricular arrhythmias was end diastolic volume index $(b=0.034, P=$ 0.012 ). However, when the same analyses were performed with any ventricular arrhythmias as a dependent variable, none of the variables was predictive of arrhythmias.

Comparison of left ventricular volumes between patients with and without complex ventricular arrhythmias at each time point showed significantly higher end diastolic volume index in patients with arrhythmias on days 2 and 3 and after three weeks (table 5 and fig 1); end systolic volume index was also higher in patients with complex ventricular

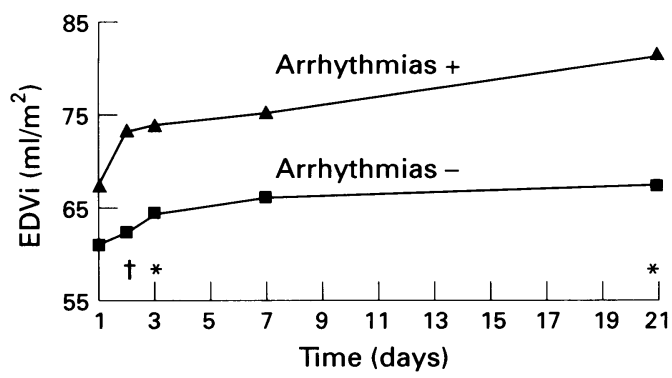

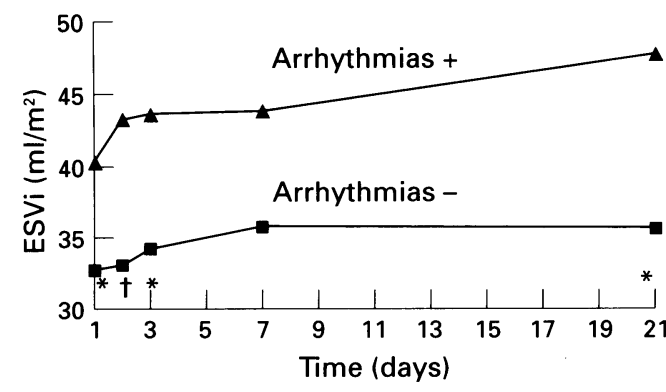

Figure 2 Association of complex ventricular arrhythmias with end systolic volume changes. Arrhythmias + , patients with complex ventricular arrhythmias; Arrhythmias -, patients without complex ventricular arrhythmias; ESVi, end systolic volume index. ${ }^{\star} P<0.05 ;+P<0.01$.

arrhythmias on day 1,2 , and 3 and after three weeks (table 5 and fig 2).

Assessment of the association of arrhythmias with left ventricular volume changes over three weeks using repeated measures analysis of variance showed that in patients with complex ventricular arrhythmias both end diastolic (fig 1) and end systolic volume indices (fig 2) were significantly higher throughout the study period $(F=5.62, P=0.046$ and $F=6.42$, $P=0 \cdot 017$, respectively).

The two stage regression model showed that both intercept and slope of end diastolic and end systolic volume indices were significantly higher in patients with complex ventricular arrhythmias compared to patients without complex arrhythmias (table 6).

\section{Discussion}

The serial echocardiography performed in this study has shown that complex ventricular arrhythmias following myocardial infarction are associated with early ventricular dilatation. Patients with arrhythmias had larger ventricular volume (diastolic and systolic) at initial examination and a significantly greater increase over the subsequent three weeks than those without complex arrhythmias.

The incidence of complex arrhythmias in this population based series of myocardial infarction was relatively low, although antiarrhythmic treatment was discontinued before Holter monitoring. This is similar to findings of Turrito et $a l^{9}(21 \%)$ and Leor et $a l^{10}$ (13-16\%), but in contrast to earlier studies reporting incidences from $30 \%$ to $80 \% .^{411}$ These differences can be attributed to different criteria that were used for classification of complex ventricular arrhythmias. Also, the lower incidence of arrhythmias in the late hospital phase detected in more recent studies may reflect improved management of myocardial infarction during the acute phase.

The relation between ventricular arrhythmias in the late hospital phase of acute myocardial infarction and sudden death after hospital discharge was well established in mid 1970s. Subsequent studies have shown that ventricular arrhythmias are usually associated with a depressed left ventricular ejection fraction, suggesting the possibility that they are not an independent prognostic factor. ${ }^{111}$ 
Although this issue was extensively investigated, the interplay between ventricular arrhythmias and left ventricular function after myocardial infarction has not been clarified yet. ${ }^{12}$

The concept of left ventricular remodelling after myocardial infarction was introduced into clinical practice by McKay et al. ${ }^{13}$ An early change in this process is infarct expansion, ${ }^{2}$ in which disruption of normal myofibrils leads to thinning and dilatation of the necrotic zone. Histological analyses have shown that thinning of the infarcted area is the result of disruption of myofibrils, leading to the decrease of myocyte number in the infarct zone. ${ }^{14}$ During the healing phase, connective tissue cells connect disrupted myofibrils, creating resistance to further elongation. ${ }^{15}$ Expansion occurs almost exclusively in patients with large transmural infarcts and may be associated with heart failure, aneurysm formation, or myocardial rupture. ${ }^{15}$ Infarct expansion can be detected by cross sectional echocardiography as an alteration of left ventricular geometry resulting from elongation of the non-contractile region. ${ }^{16}$

It has been shown that measurement of the ejection fraction is not an ideal method for assessing left ventricular systolic function after certain therapeutic interventions in patients with myocardial infarction. ${ }^{17}$ Acute myocardial infarction is associated with akinesis or dyskinesis of the infarcted area, which leads to compensatory hyperkinesis of the remaining segments in order to maintain stroke volume and ejection fraction. White et $a l^{18}$ have shown that although survival is directly related to ejection fraction, end systolic volume is the most important predictor of future mortality.

\section{LEFT VENTRICULAR REMODELLING}

With these observations in mind, we investigated the relation between left ventricular remodelling and ventricular arrhythmias. It appeared that complex ventricular arrhythmias were indeed related to early left ventricular dilatation (presumably due to infarct expansion) rather than to a poor ejection fraction. This was shown by several different analyses used in this study. With logistic regression modelling we found that the only variables predictive of arrhythmias were left ventricular end diastolic and end systolic volume indices, either determined on admission or in the late hospital phase. Repeated measures analysis of variance has confirmed that complex ventricular arrhythmias are strongly associated with the increase of left ventricular volume throughout the study period. Finally, temporal analysis has shown that initial volumes were higher and subsequent end diastolic and end systolic volume changes more pronounced in patients with complex ventricular arrhythmias.

Experimental studies ${ }^{19}$ have demonstrated that myocardial stretch can initiate ventricular arrhythmias and that susceptibility to stretch induced arrhythmias is enhanced by ventricular dilatation. Recently, Søgaard et al ${ }^{20}$ have assessed the effect of captopril on ventricular arrhythmias and left ventricular remodelling; to our knowledge, this is the only study that has investigated the relation between arrhythmias and ventricular remodelling in a manner similar to ours. They found that the end diastolic volume index was highly predictive of arrhythmias, but were not able to show the same for end systolic volume index. However, in our study, end systolic volume index on admission was the best predictor of arrhythmias in the late hospital phase. These findings may have several important clinical implications. First, since it has been shown that left ventricular enlargement after myocardial infarction is associated with decreased survival from congestive heart failure ${ }^{21}$ and that the risk of death is directly related to left ventricular size ${ }^{22}$ it may be hypothesised that late mortality in these patients is partly related to complex arrhythmias, not only to heart failure. Second, they suggest that determination of left ventricular volume early after myocardial infarction may predict complex arrhythmias in the late hospital phase or after hospital discharge. Third, it may be hypothesised that prevention of infarct expansion (by establishing the patency of the infarct related artery ${ }^{23}$ or by early use of ACE inhibitors) may prevent not only left ventricular remodelling, but also complex ventricular arrhythmias.

Théroux et al ${ }^{24}$ have shown that patients with larger infarcts, that is, with more severe wall motion abnormalities (akinesis or dyskinesis), are more likely to have ventricular arrhythmias than patients with normal wall motion or hypokinesis; however, creatine kinase values were not reported in this study. Our results suggest that infarct size, as assessed by peak creatine kinase values was larger in patients with complex ventricular arrhythmias. These findings indicate again that large infarcts, leading to infarct expansion and subsequent ventricular dilatation, are more likely to be associated with complex ventricular arrhythmias.

\section{STUDY LIMITATIONS}

The major limitation of the study is the small number of patients studied and the small number of patients with complex ventricular arrhythmias. It might also be questioned why Lown class 3 to 5 was used to define complex ventricular arrhythmias. It was felt that postinfarct patients with complex arrhythmias are at increased risk of sudden death and may be considered for antiarrhythmic treatment, ${ }^{25}$ which would not be the case for patients with isolated premature ventricular contractions (Lown class 1 and 2), no matter how frequent. Also, since the occurrence of ventricular arrhythmias after myocardial infarction may vary from day to day, a single Holter monitor may not be representative of the true incidence of arrhythmias; electrophysiological studies would be likely to have provided more accurate insight into the relation between potentially lethal ventricular arrhythmias and left ventricular remodelling, but this was not practical in our study. However, despite these limitations, we believe this large series of consecutive patients with serial volume data 
makes this study unique in comparison to previous ones.

\section{CONCLUSIONS}

Our study indicates that complex ventricular arrhythmias after myocardial infarction are associated with larger infarcts and that they are related to early left ventricular dilatation rather than to depressed ejection fraction. This supports the previous observation that left ventricular volume is the most powerful prognostic predictor after myocardial infarction. It appears that complex ventricular arrhythmias may be an aetiological factor linking left ventricular remodelling with higher mortality. However, larger follow up studies of patients with progressive left ventricular dilatation after myocardial infarction are necessary to answer these questions.

We thank Eric Topol, MD, FACC for reviewing the manuscript. This paper was presented in part at the 44th Annual Scientific Session of the American College of Cardiology, New Orleans, March 19-22, 1995 and at the 17th Congress of the European Society of Cardiology, Amsterdam, August 20-24, 1995.

1 Bigger JT, Fleiss JL, Kleiger R, Miller JP, Rolnitzky LM and The Multicenter Post-Infarction Research Grou The relationships among ventricular arrhythmias, left ventricular dysfunction and mortality in the 2 years after myocardial infarction. Circulation 1984;69:250-8.

2 Hutchins GM, Bulkley BH. Infarct expansion versus extension: two different complications of acute myocardial infarction. Am ₹ Cardiol 1978;41:1127-32.

3 Popović AD, Nešković AN, Babić R, Obradović V, Bozinovic LJ, Marinković J, et al. Independent impact of thrombolytic therapy and vessel patency on left ventricular dilatation after myocardial infarction: serial echocar-

Circulation 1994;90:800-7.

4 Schulze RA, Strauss HW, Pitt B. Sudden death in the year following myocardial infarction. Relation to ventricular premature contractions in the late hospital phase and left premature contractions in the late hospital phase and lef

5 Schiller N, Shah PM, Crawford M, DeMaria A, Devereux $\mathrm{R}$, Feigenbaum $\mathrm{H}$, et al. Recommendations for quantitation of the left ventricle by two-dimensional echocardiog raphy. $\mathcal{F}$ Am Soc Echocardiogr 1989;2:358-68.

6 Lown B, Wolf $M$. Approaches to sudden death from coronary heart disease. Circulation 1971;44:130-42.

7 The TIMI study group: The thrombolysis in myocardia infarction (TIMI) trial. Phase I findings. $N$ Engl $\mathcal{F}$ Med 1985;312:932-6.

8 Laird NM, Ware JH. Random effects models for longitudinal data. Biometrics 1982;38:963-74.

9 Turrito G, Risa AL, Zanchi E, Prati PL. The signal aver- aged electrocardiogram and ventricular arrhythmias after thrombolysis for acute myocardial infarction. $7 \mathrm{Am} \mathrm{Coll}$ Cardiol 1990;15:1270-6.

10 Leor J, Hod H, Rotstein Z, Truman S, Gansky S, Goldbourt U, et al. Effects of thrombolysis on the 12-lead signal-averaged ECG in the early postinfarction period. Am Heart $\mathcal{1}$ 1990;120:495-502.

11 Olson HG, Lyons KP, Troop P, Butman SM, Piters KM. Prognostic implications of complicated ventricular arrhythmias early after hospital discharge in acute arrhythmias early after hospital discharge in acute myocardial infarction: A serial ambulatory elect

12 Juul-Möller S, Lilja B, Johansson BW. Ventricular arrhythmias and left ventricular function: one year follow-up after myocardial infarction. Eur Heart f 1988;9:1181-7.

13 McKay RG, Pfeffer MA, Pasternak RC, Markis JE, Come PC, Nakao S, et al. Left ventricular remodeling after myocardial infarction: a corollary to infarct expansion. Circulation 1986;74:693-702.

14 Weisman HF, Bush DE, Mannisi JA, Weisfeldt ML, Healy B. Cellular mechanisms of myocardial infarct expansion. Circulation 1988;78:186-201.

15 Schuster EH, Bulkley BH. Expansion of transmural myocardial infarction: a pathophysiologic factor in cardiac rupture. Circulation 1979;60:1532-8.

16 Erlebacher JA, Weiss JL, Eaton LW, Kallman C, Weisfeldt $\mathrm{ML}$, Bulkley $\mathrm{BH}$. Late effects of acute infarct dilatation on heart size: a two-dimensional echocardiographic study. Am 7 Cardiol 1982;49:1120-6.

17 Topol E. Thrombolytic intervention, In: Topol E, ed. Textbook of interventional cardiology. Philadelphia: WB Saunders, 1994:75-7.

18 White HD, Norris RM, Brown MA, Brandt PWT, Whitlock RML, Wild CJ. Left ventricular end-systolic Whitlock RML, Wild CJ. Left ventricular end-systolic
volume as the major determinant of survival after recovery volume as the major determinant of survival after recovery

19 Hansen DE, Craig CS, Hondeghem LM. Stretch-induced arrhythmias in the isolated canine ventricle. Evidence for the importance of mechanoelectrical feedback. Circulation 1990;81:1094-105.

20 Søgaard P, Gøtzsche CO, Ravkilde J, Nørgaard A Thygesen $K$. Ventricular arrhythmias in the acute and chronic phases after acute myocardial infarction. Effect of intervention with captopril. Circulation 1994;90:101-7.

21 Multicenter Postinfarction Research Group. Risk stratifica tion and survival after myocardial infarction. $N$ Engl $f$ Med 1983;309:331-6.

22 Pfeffer MA, Pfeffer JM. Ventricular enlargement and reduced survival after myocardial infarction. Circulation 1987;75(suppl 4):IV-93-7.

23 Popović AD, Nešković AN, Marinković J, Thomas JD. Acute and long-term effects of thrombolysis after anterior wall acute myocardial infarction with serial assessment of infarct expansion and late ventricular remodeling. $A m \mathcal{F}$ infarct expansion and late
Cardiol 1996;77:446-50.

24 Théroux $P$, Morissette D, Juneau M, de Guise P, Pelletier $G$, Waters DD. Influence of fibrinolysis and percutaneous transluminal coronary angioplasty on the frequency of ventricular premature complexes. $\mathrm{Am} \mathcal{F}$ Cardiol 1989;63:797-801.

25 Burkart F, Pfifester M, Kiowski W, Follath F, Burckhardt $D$. Effect of antiarrhythmic therapy on mortality in survivors of myocardial infarction with asymptomatic complex ventricular arrhythmias: Basel Antiarrhythmic Study of Infarct Survival (BASIS). 7 Am Coll Cardiol 1990;16: $1711-8$ 